\title{
Fired up, not burnt out - focusing on the rewards of working in psychiatry
}

\author{
S. Priebe ${ }^{1}$ and U. Reininghaus ${ }^{1,2}$ \\ ${ }^{1}$ Unit for Social and Community Psychiatry, Barts and the London School of Medicine, Queen Mary University of London, London, UK \\ ${ }^{2}$ Department of Psychiatry, University of Cambridge, Cambridge, UK
}

A positive morale of staff is essential for the quality of mental health care. There are at least two reasons for this. One is the impact on recruitment and retention. The current difficulties to recruit and retain sufficient numbers of qualified staff vary across countries and even within the same country across different professional groups. For example, they can be markedly different for psychiatrists, clinical psychologists and psychiatric nurses. However, in many countries, the difficulties of filling posts with qualified staff are significant, and problems of attracting talented young medical graduates into psychiatry are wide spread. Without qualified staff there is no qualified mental health care. Alongside other factors such as salary, working conditions and social prestige, a high staff morale is likely to lure more people into the profession and make them continue to work in it. Another reason is the influence on treatment outcomes for patients. Staff not only need expertise, competence and skills but also need good morale to deliver the best possible care that leads to positive results. Evidence suggests that a better morale in community mental health care leads to more favourable patient outcomes (Priebe et al., 2004).

\section{Stress and burnout}

For improving staff morale and maintaining it at the highest possible level, a concept is required for how to understand and influence morale. The dominating concept in the literature has been that of stress and burnout (Maslach and Jackson, 1981; Maslach et al. 1996). In a simplified summary of the concept, negative external factors coupled with unhelpful attitudes and less than optimal coping strategies may lead to distress and burnout of staff. The risk factors for such negative processes need to be identified and prevented. Research informed by this concept has produced interesting and valuable results. Stress and

Address for correspondence: Professor Stefan Priebe, Unit for Social and Community Psychiatry, Barts and the London School of Medicine, Queen Mary University of London, Newham Centre for Mental Health, London E13 8SP, United Kingdom.

(E-mail: S.Priebe@qmul.ac.uk) burnout can be measured, and existing scales can identify significant differences even in relatively small samples (Reininghaus \& Priebe, 2007). A range of intervention programmes directed at individuals and organisations have been developed and tested. Although most studies have been conducted with professionals outside mental health care, the conclusion of a recent review that intervention programs against burnout are beneficial' (Awa et al., 2010, p. 184) may be assumed to apply also to mental health professionals. So far, so good.

\section{Limitations of the concept}

Although the concept of stress and burnout has obvious merits, it also has limitations and possibly even unhelpful implications.

In the stress model, all experience is potential stress, usually of a negative kind, although the notion of a positive eu-stress has also been raised (Selye, 1975). According to the model, stress impacts on staff, and staff can have different degrees of resilience and strengths to withstand the stress (Rutter, 1985). If they withstand the pressure and negative influences with their resilience, fine. If they do not, there will be distress and suffering. This is a very mechanical model reflecting the stress put on physical material. Such a model may be insufficient to capture the complexity and potentials of social and professional life. To illustrate this, we take an example for an important aspect of life that is (usually) not professional: love. Being in love is not just positive or negative stress that we need to cope with, but a key experience in life with various enjoyable and less enjoyable aspects and a consistently changing whole of experiences, emotions and thoughts. The mechanical stress model appears insufficient to reflect the fascination and deep emotions that can be part of love. This may also apply to the complex experience of working in mental health care, and the stress model cannot fully reflect the unusual and often rich experience of working in mental health care which in most settings comes with a range of different and sometimes surprising human encounters. 
One might argue that any concept has its limitations, but this does not make it detrimental. Why should the concept of stress and burnout be potentially harmful? In an occupational context, the term stress tends to be linked with some blame, commonly of the working conditions for which the employer and not the employee is responsible. Stress and burnout need to be prevented, and this is often seen as being beyond the influence and responsibility of the individual and in the realm of a higher authority. It leads to accusing others, be it individuals or organisations, who should change the situation and improve working conditions, while staff themselves are passive subjects of stress. This tendency may have been supported by an Anglo-American culture of litigation (it is interesting to note that the stress model has become particularly popular within that culture) and is in line with a generally exculpating and defensive mindset. Such a mindset is not helpful to actively influence the working context and bring about improvements. On the contrary, the resulting tendency to complain and feel unfairly treated can stand in the way of positive action and be non-constructive. Specific complaints and burnout scores may be misleading. For example, in a study among mental health professionals in Austria, those with the highest burnout levels already had the lowest caseloads (Swoboda et al., 2006). In a comparison of six professional groups, i.e. psychiatrists, nurses and social workers each in London and Berlin, only one of the six groups frequently complained about low pay as a major problem in their job. This group were the psychiatrists in London, who in objective terms had by far the highest salary of all six groups (Priebe et al., 2005). Whatever might have improved the morale of the interviewed psychiatrists in London, it is unlikely that an even further increased salary gap between them and the other five non-complaining groups would have been the most effective way forward. A focus on stress and burnout may feed into a defensive, complaining and complacent attitude.

\section{Work engagement}

There is an alternative to the rather negative stress model. It is related to the recent rise of positive psychology (Seligman \& Csikszentmihalyi, 2000). The traditional bias of psychology towards negative experiences is reflected by the ratio of publications on negative states to positive states which has been reported as 14:1 (Myers, 2000). Positive psychology has shifted the focus from trying to prevent or repair bad things in life to building positive qualities. It has been suggested that only a focus on the positive sides of work may help to gain a full understanding of the meaning and effects of working. Bakker et al. (2008) called for a focus on 'work engagement: a positive, fulfilling, effective-motivational state of work-related well-being that can be seen as the antipode of job burnout' (Bakker et al., 2008, p. 188). Work engagement is associated with high levels of energy and enthusiasm about work. Research has shown that work engagement is indeed at the opposite end of burnout on core factors describing the work experience (Gonzalez-Roma et al., 2006). Thus, work engagement can still capture aspects that may prevent or alleviate burnout, but takes a more positive and arguably more comprehensive perspective.

\section{Mental health care}

Working in mental health care provides a wide range of experiences, relationships and encounters not only with all risks for frustration and disappointment but also with joy, fascination and satisfaction. There may be no single aspect of working in mental health care that makes it absolutely unique. Yet, the depth of relationships, the range of different encounters, the options to engage with different people and the challenge to analyse and deal with complex situations on a personal and also societal level are certainly unusual and may make it attractive. In the surveys among professionals, positive relationships with patients and the diversity and challenge of the work were reported as the most enjoyable aspects of work (Galeazzi et al., 2004). As important as science and evidence-based medicine are for psychiatry, they are not the full picture of the attraction of working in psychiatry. It is the human encounters, the challenge that often is linked with uncertainty, and the positive emotional experience that can make the jobs attractive, ever changing and fulfilling.

There is another reason - specifically for mental health care - for focusing on positive aspects of the profession rather than stress and burnout. The attitude of professional groups is likely to be reflected in their relationships with patients. For a number of reasons, clinicians should help patients to discover, develop and utilise their strengths rather than only contemplating deficits and problems. Conveying a positive perspective has been emphasised in a number of therapeutic models throughout the history of psychiatry. It is more likely to be achieved if clinicians themselves take a similarly positive perspective of their role and work.

We conclude that the challenge is to fire up professionals in mental health care rather than prevent their burnout. For this, a stronger focus on the rewards of working in psychiatry is required, and we believe that 
the rewards can be substantial and unusual. It may be time to study the positive aspects more extensively and consider them in attempts to improve staff morale.

\section{References}

Awa W, Plaumann M, Walter U (2010). Burnout prevention: a review of intervention programs. Patient Education and Counseling 78, 184-190.

Bakker A, Schaufeli W, Leiter M, Taris T (2008). Work engagement: an emerging concept in occupational health psychology. Work and Stress 22, 187-200.

Galeazzi G, Delmonte S, Fakhoury W, Priebe S (2004). Morale of mental health professionals in community mental health services of a Northern Italian province. Epidemiologia e Psichiatria Sociale 13, 191-197.

Gonzalez-Roma V, Schaufeli W, Bakker A, Lloret S (2006). Burnout and engagement: independent factors or opposite poles? Journal of Vocational Behavior 68, 165-174.

Maslach C, Jackson S (1981). Maslach Burnout Inventory Manual (Human Services Survey). Consulting Psychologists Press: Menlo Park, CA.

Maslach C, Jackson S, Leiter M (1996). Maslach Burnout Inventory Manual, 3rd edn. Consulting Psychologists Press: Menlo Park, CA.

Myers D (2000). The funds, friends and faith of happy people. American Psychologist 55, 56-67.
Priebe S, Fakhoury W, White I, Watts J, Bebbington P, Billings J, Burns T, Johnson S, Muijen M, Ryrie I, Wright C (2004). Characteristics of teams, staff and patients: associations with outcomes of patients in assertive outreach. British Journal of Psychiatry 185, 306-311.

Priebe S, Fakhoury W, Hoffmann K, Powel R (2005). Morale and job perception of community mental health professionals in Berlin and London. Social Psychiatry and Psychiatric Epidemiology 40, 223-232.

Reininghaus U, Priebe S (2007). Assessing morale in community mental health professionals: a pooled analysis of data from four European countries. Social Psychiatry and Psychiatric Epidemiology 42, 237-243.

Rutter M (1985). 'Resilience in the face of adversity. Protective factors and resistance to psychiatric disorder'. British Journal of Psychiatry 147, 598-611.

Seligman M, Csikszentmihalyi M (2000). Positive psychology: an introduction. American Psychologist 55, 5-14.

Selye H (1975). Confusion and controversy in the stress field. Journal of Human Stress 1, 37-44.

Swoboda H, Sibitz I, Frühwald S, Klug G, Bauer B, Priebe S (2006). Wie erleben Professionelle der gemeindepsychiatrischen Versorgung in Österreich ihre Tätigkeit? - Eine inhaltsanalytische Studie. (How do community-based mental health staff in Austria perceive their job? - A qualitative study.) Psychiatrische Praxis 22, $67-73$ 